\title{
2- Input AND Gate For Fast Gating and Switching Based On XGM Properties of InGaAsP Semiconductor Optical Amplifier
}

\author{
Vikrant k Srivastava \\ Electronics \& Instrumentation \\ Department \\ Indian School of Mines \\ Dhanbad 826004 ,INDIA. \\ srivastavavikrant@hotmail.com
}

\author{
Devendra Chack \\ Electronics \& Communication Deptt \\ BCT- Kumaon engineering College \\ Dwarahat Almora UK \\ 263653 ,INDIA. \\ devendra.chack@gmail.com
}

\author{
Vishnu Priye \\ Electronics \& Instrumentation \\ Department \\ Indian School of Mines \\ Dhanbad 826004 ,INDIA. \\ vpriye@gmail.com
}

\begin{abstract}
We report on an all-optical AND-gate using simultaneous Four-Wave Mixing (FWM) and Cross-Gain Modulation (XGM) in a semiconductor optical amplifier (SOA). The operation of the proposed AND gate is simulated and the results demonstrate its effectiveness. This AND gate could provide a new possibility for all-optical computing and all-optical routing in future all-optical networks. In an AND ( AB ) gate, Boolean is firstly obtained by using signal $B$ as a pump beam and clock signal as a probe beam in SOA-1. By passing signal A as a probe beam and as a pump beam through SOA-2, Boolean $A B$ is acquired. Proposed optical logic unit is based on coupled nonlinear equations describing XGM and FWM effects. These equations are first solved to generate the pump, probe and conjugate pulses in a SOA. The pulse behavior are analyzed and applied to realize behavior of all-optical AND gate and its function is verified with the help of waveform and analytical assumption.
\end{abstract}

Keywords: Optical Logic Gates, Semiconductor Optical Amplifier, SOA, Four Wave Mixing, Cross Gain Modulation

\section{INTRODUCTION}

A lot of schemes based on cross-gain modulation (XGM) have been reported, such as AND gates [1], NAND gate [2], NOR gates [3, 4], XOR gate [5] etc. Cross-gain modulation (XGM), one of several wavelength technique methods based on SOAs, is simple to implement and has shown impressive operation for high bit rates [6-8]. Electrons in the SOA are placed in the excited states when electrical current is applied to an SOA. An incoming optical signal stimulates the excited electrons, and settled to the ground states after the signal is amplified. This stimulated emission continues as the input signal travels through the SOA until the photons exit together as an amplified signal. However, the amplification of the input signal consumes carriers thereby transiently reducing a gain, which is called gain saturation. The carrier density changes in an SOA will affect all of the input signals, so it is possible that a signal at one wavelength affect the gain of signal at another wavelength. This nonlinearity property is called XGM based on an SOA. When a pulse exists for the pump signal passing through an SOA, it causes carrier depletion in the SOA. The carrier depletion leads to gain saturation in the SOA causing the marked intensity reduction of an incoming probe signal.
Therefore, the marked intensity reduction of the probe signal in the SOA leads to no pulse existence for output signal. When a pulse does not exist for the pump signal, there is no effect on the gain of probe signal in the SOA. Therefore, output signal has the same pulse as the probe signal.

In this paper the same effect is analyzed properly and then applied to get the desired result.

\section{SIMULATION METHOD}

In our approach the reference equations are taken from Ref. [9] and different parameters which are taken into consideration are tabulated below in Table I. It is assumed that input pump, and probe pulses have the same temporal width as well as perfect pulse overlap, and in all of the cases, their powers are set to a ratio of 10:1. Numerical simulations have been undertaken to investigate the amplification of strong picosecond optical pulses in semiconductor optical amplifiers (SOAs), taking into account carrier heating, spectral holeburning, carrier-carrier scattering (CCS) and carrier photon scattering (CPS). The result of interference of two copolarized pulses when propagating into SOA,one pump pulse at central frequency $\omega 1$ and the other probe pulse at central frequency $\omega 0$,induce a bit of carrier density pulsation at the frequency detuning $\Omega=\omega 1-\omega 0$. This results a generation of a new frequency pulse at $\omega 2=\omega 0-\Omega=2 \omega 0-\omega 1$. The new pulse is phase conjugate replica of the probe pulses, and can be extracted from the input pulses using an optical filter. Here , $A_{j}(Z, t), j=0,1,2$, correspond to the slowly varying envelopes of the pump, the probe, and the conjugate pulses, respectively, and $\Omega=\omega 1-\omega 0$, is the frequency detuning. [9].

The following Equation has been taken into consideration

$$
\mathrm{A}_{0}(\mathrm{~L}, \mathrm{t})=\mathrm{A}_{0}(0, \mathrm{t}) e^{\left(\frac{1}{2}\right)(1-i \alpha) \mathrm{h}}
$$

Where 
$\mathrm{A}_{0}(0, \mathrm{t})$, is the input pump pulse amplitude at any end of SOA,

$\mathrm{A}_{0}(\mathrm{~L}, \mathrm{t})$, is input pump pulse amplitude at any length $\mathrm{L}$ of SOA,

$\mathrm{L}$ is length of SOA. $\mathrm{t}$ is time . Rest parameters are already defined in Table I.

$$
\begin{aligned}
& A_{1}(L, t)=A_{1}(0, t) e^{\frac{1}{2}\left[(1-i \alpha) h-\eta_{10}\left|A_{0}(0, t)\right|^{2}\left(e^{h}-1\right)\right]} \\
& \times \cosh \left[\left(\frac{1}{2}\right) \sqrt{\eta_{02} \eta_{01}^{*}}\left|A_{0}(0, t)\right|^{2}\left(e^{h}-1\right)\right]
\end{aligned}
$$

Where SOA.

$\mathrm{A}_{1}(0, \mathrm{t}), \quad$ is input probe pulse amplitude at any end of

$\mathrm{A}_{1}(\mathrm{~L}, \mathrm{t})$, is input probe pulse amplitude at any length $\mathrm{L}$ of SOA.

$\mathrm{L}=$ length of SOA. $\mathrm{t}$ is time. Rest parameters are already defined in Table I.

$$
\begin{aligned}
& A_{2}(L, t)=\frac{-A_{1}^{*}(L, t) A_{0}(L, t)}{A_{0}^{*}(L, t)} \sqrt{\frac{\eta_{01}}{\eta_{02}^{*}}} \\
& \times \sinh \left[\left(\frac{1}{2}\right) \sqrt{\eta_{01} \eta_{02}^{*}}\left|A_{0}(L, t)\right|^{2} e^{-h}\left(e^{h}-1\right)\right]
\end{aligned}
$$

Where SOA.

$\mathrm{A}_{2}(0, \mathrm{t})$ is input conjugate pulse amplitude at any end of

$\mathrm{A}_{2}(\mathrm{~L}, \mathrm{t})$ is input conjugate pulse amplitude at any length $\mathrm{L}$ of SOA.

$\mathrm{L}=$ length of SOA, $\mathrm{t}$ is time. Rest parameters are already defined in Table I.

$$
\eta_{01}=\eta_{01}^{\mathrm{CD}}+\eta_{01}^{\mathrm{CH}}+\eta_{01}^{\mathrm{SHB}}
$$

Where,

$$
\begin{aligned}
& \eta_{01}^{\mathrm{CD}}=\varepsilon_{\mathrm{cd}} \frac{1-\mathrm{i} \alpha}{\left(\left(1+\mathrm{i} \Omega \tau_{1}\right)+\left(1+i \Omega \tau_{\mathrm{g}}\right)\right)} \\
& \eta_{01}^{\mathrm{CH}}=\varepsilon_{\mathrm{t}} \frac{1-\mathrm{i} \alpha_{\mathrm{T}}}{\left(\left(1+\mathrm{i} \Omega \tau_{\mathrm{h}}\right)+\left(1+i \Omega \tau_{1}\right)\right)} \\
& \eta_{01}^{\mathrm{SHB}}=\varepsilon_{\mathrm{shb}} \frac{1-i \alpha_{\mathrm{shb}}}{1+\mathrm{i} \Omega \tau_{1}}
\end{aligned}
$$

The amplification function $\mathrm{h}$ and coupling coefficient $\eta_{i j}$ are defined in [9]. The effects of carrier depletion, carrier heating, spectral hole burning, two-photon absorption, and ultrafast nonlinear refraction are taken into account, leading to a successful description of wave mixing for optical pulses with

\begin{tabular}{|c|c|c|c|}
\hline Parameters & Symbols & Values & Unit \\
\hline $\begin{array}{l}\text { Length of the } \\
\text { amplifier }\end{array}$ & $L$ & 450 & $\mu m$ \\
\hline Small signal gain & $G$ & $1.54 * 10^{-4}$ & $m^{-1}$ \\
\hline Carrier lifetime & $\tau_{\mathrm{s}}$ & 300 & $p s$ \\
\hline $\begin{array}{l}\text { Nonlinear gain } \\
\text { compression for } \\
\text { carrier heating }\end{array}$ & $\varepsilon_{\mathrm{t}}$ & 0.13 & $\mathrm{w}^{-1}$ \\
\hline $\begin{array}{l}\text { Non linear gain } \\
\text { compression for } \\
\text { spectral hole } \\
\text { burning }\end{array}$ & $\varepsilon_{\mathrm{shb}}$ & 0.07 & $\mathrm{w}^{-1}$ \\
\hline $\begin{array}{l}\text { Traditional } \\
\text { linewidth } \\
\text { enhancement factor }\end{array}$ & $\alpha$ & 5.0 & \\
\hline $\begin{array}{l}\text { Temperature } \\
\text { linewidth } \\
\text { enhancement factor }\end{array}$ & $\alpha_{\mathrm{T}}$ & 3.0 & \\
\hline $\begin{array}{l}\text { Linewidth } \\
\text { enhancement factor } \\
\text { for spectral hole } \\
\text { burning }\end{array}$ & $\alpha_{\mathrm{shb}}$ & 0.1 & \\
\hline $\begin{array}{l}\text { Time for carrier- } \\
\text { carrier scattering }\end{array}$ & $\tau_{1}$ & 50 & fs \\
\hline $\begin{array}{l}\text { Time for carrier } \\
\text { photon scattering }\end{array}$ & $\tau_{\mathrm{h}}$ & 700 & fs \\
\hline $\begin{array}{l}\text { Carrier depletion } \\
\text { coefficient }\end{array}$ & $\varepsilon_{\mathrm{cd}}$ & 47 & $\mathrm{w}^{-1}$ \\
\hline
\end{tabular}

strong pulse energy, and/or with pulsewidths larger than several hundred femtoseconds, as well as in active or passive optical waveguides. The parameters $\alpha, \tau$, and $\varepsilon$ determine the strength and nature of the wave mixing process caused by each of the intraband mechanisms and their relative significance. The values of $\alpha, \tau$, and $\varepsilon$ cannot be determined unanimously.

TABLE I: PARAMETERS USED IN SIMULATION WORK

\section{RESULTS AND DISCUSSION}

Truth table for AND Logic can be given as

\begin{tabular}{|l|l|l|}
\hline $\mathbf{A}$ & $\mathbf{B}$ & $\mathbf{A B}$ \\
\hline 0 & 0 & 0 \\
\hline 0 & 1 & 0 \\
\hline 1 & 0 & 0 \\
\hline 1 & 1 & 1 \\
\hline
\end{tabular}

Column $\mathrm{AB}$ of the above truth table indicates logic behaviour of AND gate. The full design needs two SOAs. 


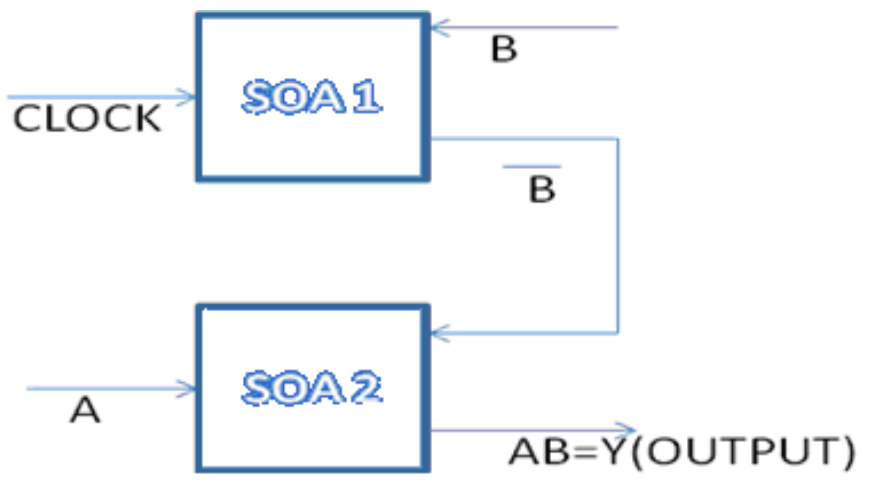

Figure 1.

Fig. 1 shows that in an AND ( $\mathrm{AB}$ ) gate, Boolean is firstly obtained by using signal B as a pump beam and clock signal as a probe beam in SOA-1. Next, by passing signal A as a probe beam and as a pump beam through SOA-2, Boolean $\mathrm{AB}$ is acquired.

The following waveforms shows different outputs of simulation of above equations.
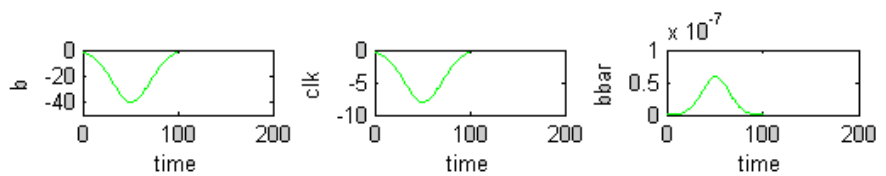

Figure 2 . ( Pump $b=0$, Probe $\mathrm{clk}=0)$
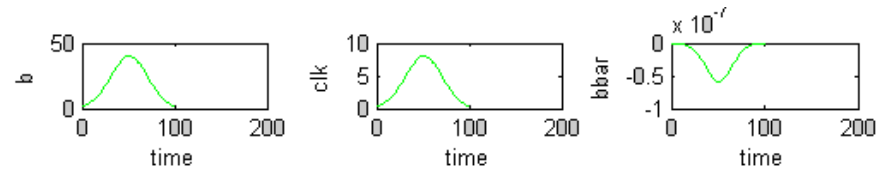

Figure 3. ( Pump $b=1$, Probe $c l k=1$ )
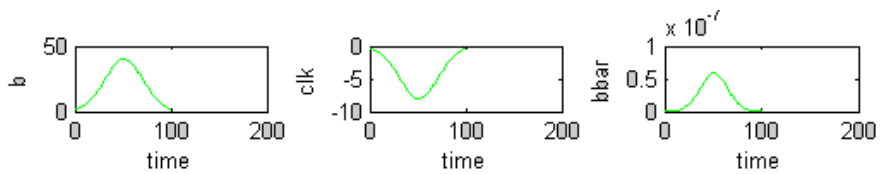

Figure 4. (Pump b=1, Probe clk=0)
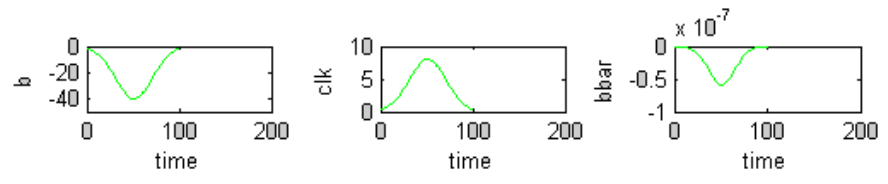

Figure 5. ( Pump $b=0$, Probe $c l k=1$ )
Analysing Fig. 2, Fig. 3, Fig. 4,and Fig.5 ,we observe an interesting result.The result shows that on proper manipulation of pump 'b', and probe 'clk'.we can get desired outputs. In Fig. 2 and Fig. 3 the input pump get inverted whereas in Fig. 4 and Fig. 5, the outputs are same as that of inputs. The generated signal when filtered out and when applied as a pump with a different probe named 'A', in SOA 2 , results the desired logic of an AND Gate.

\section{CONCLUSIONS}

In this paper, it is shown that using analytical solutions of nonlinear effects in semiconductor optical amplifier, we can model an AND Circuit. This research has guided readers to design all-optical AND logic circuits so that anyone can construct any types of all-optical different logic circuits by utilizing the detailed process of designing the AND Gate..

\section{REFERENCES}

[1] X. Zhang, Y. Wang, J. Sun, D. Liu, and D. Huang, "All-optical AND gate at $10 \mathrm{Gbit} / \mathrm{s}$ based on cascaded single-port-couple SOAs," Opt. Express 12, 361-366 (2004).

[2] S.H. Kim, J.H. Kim, B.G. Yu, Y.T. Byun, Y.M. Jeon, S. Lee, D.H. Woo, and S.H. Kim, "All-optical NAND gate using cross-gain modulation in semiconductor optical amplifiers," Electronics Letters 41, 10271028(2005).

[3] A. Hamie, A. Sharaiha, M. Guegan, and B. Pucel, "All-optical logic NOR gate using two-cascaded semiconductor optical amplifiers," IEEE Photon. Technol. Lett. 14, 1439-1441(2002) .

[4] C. Zhao, X. Zhang, H. Liu, D. Liu, and D. Huang, "Tunable all-optical NOR gate at $10 \mathrm{~Gb} / \mathrm{s}$ based on SOA fiber ring laser," Opt. Express 13, 2793-2798 (2005).

[5] J.H. Kim, Y.M. Jhon, Y.T. Byun, S. Lee, D.H. Woo, and S. H. Kim, "Alloptical XOR gate using semiconductor optical amplifiers without additional input beam," IEEE Photon. Technol. Lett. 14, 1436 1438(2002).

[6] Kim, J.H., et al.: 'All-optical XOR gate using semiconductor optical amplifiers without additional input beam', IEEE Photonics Technol. Lett.,2002, 14, pp. 1436-1438

[7] Kim, J.H., et al.: 'All-optical AND gate using cross-gain modulation in semiconductor optical amplifiers', Jpn. J. Appl. Phys., 2004, 43, pp. 608-610

[8] Ellies, A.D., et al.: 'Error free $100 \mathrm{Gbit}=\mathrm{s}$ wavelength conversion using grating assisted cross-gain modulation in $2 \mathrm{~mm}$ long semiconductor optical amplifier', Electron. Lett., 1998, 34, pp. 1958-1959

[9] S Junqlang et al., "Analytical solution of Four wave mixing between picosecond opticalpulses in semiconductor optical amplifiers with cross gain modulation and probedepletion", Microwave and Optical technology Letters, Vol. 28, No. 1, pp 78 - 82, 2001.

[10] Chan, L.Y., Qureshi, K.K., Wai, P.K.A., Moses, B., Lui, L.F.K., Tam, H.Y., Demokan, M.S.: 'All-optical bit-error monitoring system using cascaded inverted wavelength converter and optical NOR gate', IEEE photonic technology letters, 2003, 15, (4), pp.593-595.

[11] Martinez, J.M., Ramos, F., Marti, J.: 'All-optical packet header processor based on cascaded SOA-MZIs', Electronics letters, 2004, 40,(14), pp.894-895

[12] G. Berrettini, A. Simi, A. Malacarne, A. Bogoni, and L. Potí, Member, IEEE:' Ultrafast Integrable and Reconfigurable XNOR, AND,NOR, and NOT Photonic Logic Gate', IEEE Photonic technology letters, Vol. 18, No. 8, april 15, 2006 